\title{
Anaplastic Astrocytoma
}

National Cancer Institute

\section{Source}

National Cancer Institute. Anaplastic Astrocytoma. NCI Thesaurus. Code C9477.

A diffusely infiltrating, WHO grade III astrocytoma with focal or dispersed anaplasia, and a marked proliferative potential. It may arise from a low-grade astrocytoma, but it can also be diagnosed at first biopsy, without indication of a less malignant precursor lesion. It has an intrinsic tendency for malignant progression to glioblastoma. (WHO) 